\title{
A relativistic action-at-a-distance description of gravitational interactions?
}

\author{
Domingo J. Louis-Martinez \\ Department of Physics and Astronomy, \\ University of British Columbia \\ Vancouver, Canada, V6T 1 Z1 \\ martinez@phas.ubc.ca
}

\begin{abstract}
It is shown that certain aspects of gravitation may be described using a relativistic action-at-a-distance formulation. The equations of motion of the model presented are invariant under Lorentz transformations and agree with the equations of Einstein's theory of General Relativity, at the first Post-Newtonian approximation, for any number of interacting point masses.
\end{abstract}

PACS No. 03.30. +p, 04.25. -g, 04.25. Nx, 04.90. +e.

Introduction

After the discovery of the action-at-a-distance formulation of electrodynamics [1] - [10], several relativistic non-instantaneous action-at-a-distance theories have been investigated [11] - 21]. Instantaneous action-at-a-distance formulations have been studied using a variety of approaches [22] - 31]. For gravity, several relativistic action-at-a-distance models have been proposed [32] - [46], and compared with observations [47, 48]. A major difficulty with most of the models proposed is their disagreement with Einstein's theory of General Relativity (GR) in the so-called "slow motion approximation" (first Post-Newtonian approximation $(1 \mathrm{PN}))$, even for the simpler case of two point masses $(N=2)$.

The objective of this paper is to present a relativistic action-at-a-distance description of gravitational interactions for a system consisting of an arbitrary number $N$ of point masses.

The model presented in this paper is in agreement with GR at 1PN for an arbitrary number $N$ of interacting point masses.

Our description also agrees with GR in the so-called "fast motion approximation" for $N$ point masses (at the first Post-Minkowskian order (1PM)) and it is in agreement with GR for the one body case $(N=1)$ at all orders (assuming the central mass is not spinning) if the Schwarzschild metric is expressed in the isotropic gauge.

An action functional for gravity in the relativistic action-at-a-distance formulation

In order to describe a relativistic system of $N$ point masses interacting gravitationally, we consider the following action functional:

$$
\begin{aligned}
S= & -\sum_{i} m_{i} c \int d \lambda_{i} \zeta_{i}+\sum_{i} \sum_{j \neq i} \frac{G m_{i} m_{j}}{c} \iint d \lambda_{i} d \lambda_{j} \delta\left(\rho_{i j}\right) F_{i j} \\
& +\sum_{i} \sum_{j \neq i} \sum_{k \neq i, j} \frac{G^{2} m_{i} m_{j} m_{k}}{c^{3}} \iiint d \lambda_{i} d \lambda_{j} d \lambda_{k} \delta\left(\rho_{i j}\right) \delta\left(\rho_{j k}\right) F_{i j k}+\ldots
\end{aligned}
$$


In (1),$m_{i}(i=1,2, \ldots, N)$ is the mass of particle $i, \lambda_{i}$ is a Poincaré invariant parameter labelling the events along the world line $z_{i}^{\mu}\left(\lambda_{i}\right)$ of particle $i, c$ is the speed of light and $G$ the universal gravitational constant.

The functions $F_{i j}=F_{i j}\left(\xi_{i j}, \gamma_{i j}, \gamma_{j i}, \zeta_{i}, \zeta_{j}\right), F_{i j k}=F_{i j k}\left(\xi_{i j}, \xi_{i k}, \xi_{j k}, \gamma_{i j}, \gamma_{j i}, \gamma_{i k}, \gamma_{k i}, \gamma_{j k}, \gamma_{k j}, \zeta_{i}, \zeta_{j}, \zeta_{k}\right)$, are invariant under Poincaré transformations since they themselves are assumed to be functions of the Poincaré invariants $\xi_{i j}, \gamma_{i j}$, and $\zeta_{i}$. The Poincaré invariants $\rho_{i j}, \xi_{i j}, \gamma_{i j}$, and $\zeta_{i}$, are defined as follows [49]:

$$
\begin{gathered}
\rho_{i j}=\left(z_{i}-z_{j}\right)^{2} \\
\xi_{i j}=\left(\dot{z}_{i} \dot{z}_{j}\right) \\
\gamma_{i j}=\left(\dot{z}_{i}\left(z_{j}-z_{i}\right)\right) \\
\zeta_{i}=\dot{z}_{i}^{2}
\end{gathered}
$$

We denote $\dot{z}_{i}^{\mu}=\frac{d z_{i}^{\mu}}{d \lambda_{i}}$. The metric tensor: $\eta_{\mu \nu}=\operatorname{diag}(+1,-1,-1,-1)$.

The action functional (11) is invariant under Lorentz transformations and does not involve any fields to mediate the interactions between the masses. The particles interact with each other directly and we assume that the interactions propagate at the speed of light $c$ in vacuum. The Dirac delta functions in (11) account for the interactions propagating at the speed of light forward and backward in time.

The action (11) can be written in a compact form as follows:

$$
\begin{aligned}
S= & -\sum_{i} m_{i} c \int d \lambda_{i} \zeta_{i} \\
& +\sum_{k=2}^{N} \sum_{i_{1}} \sum_{i_{2} \neq i_{1}} \ldots \sum_{i_{k} \neq i_{1}, \ldots, i_{k-1}} \frac{G^{k-1} m_{i_{1}} \ldots m_{i_{k}}}{c^{2 k-3}} \int \ldots \int d \lambda_{i_{1}} \ldots d \lambda_{i_{k}} \prod_{l=1}^{k-1} \delta\left(\rho_{i_{l} i_{l+1}}\right) F_{i_{1} \ldots i_{k}}
\end{aligned}
$$

Notice that not only the two-body interactions $(k=2)$, but all possible $\mathrm{k}$-body interactions $(k=2, \ldots, N)$ contribute to the action.

Without loss of generality we can assume that $F_{j i}=F_{i j}, F_{k j i}=F_{i j k}$ and so on $\left(F_{i_{k} i_{2} \ldots i_{k-1} i_{1}}=\right.$ $\left.F_{i_{1} i_{2} \ldots i_{k-1} i_{k}}\right)$.

From (1), we can see that we can write the action of an individual particle $i$ as follows:

$$
\begin{aligned}
& S_{i}=-m_{i} c \int d \lambda_{i}\left(\zeta_{i}-\frac{2 G}{c^{2}} \sum_{j \neq i} m_{j} \int d \lambda_{j} \delta\left(\rho_{i j}\right) F_{i j}\right. \\
& \left.-\frac{G^{2}}{c^{4}} \sum_{j \neq i} \sum_{k \neq i, j} m_{j} m_{k} \iint d \lambda_{j} d \lambda_{k}\left(\delta\left(\rho_{i j}\right) \delta\left(\rho_{j k}\right) F_{i j k}+\delta\left(\rho_{j k}\right) \delta\left(\rho_{k i}\right) F_{j k i}+\delta\left(\rho_{k i}\right) \delta\left(\rho_{i j}\right) F_{k i j}\right)+\ldots\right)
\end{aligned}
$$

The equations of motion of the relativistic particles can be derived from the action (1) (or from (7)) using the variational principle. We find: 


$$
\begin{aligned}
& \ddot{z}_{i}^{\mu}+\frac{G}{c^{2}} \sum_{j \neq i} m_{j} \int d \lambda_{j}\left(\frac{\partial}{\partial z_{i \mu}}\left(\delta\left(\rho_{i j}\right) F_{i j}\right)-\frac{d}{d \lambda_{i}}\left(\delta\left(\rho_{i j}\right) \frac{\partial F_{i j}}{\partial \dot{z}_{i \mu}}\right)\right) \\
& +\frac{G^{2}}{2 c^{4}} \sum_{j \neq i} \sum_{k \neq i, j} m_{j} m_{k} \iint d \lambda_{j} d \lambda_{k}\left(\delta\left(\rho_{j k}\right)\left(\frac{\partial}{\partial z_{i \mu}}\left(\delta\left(\rho_{i j}\right) F_{i j k}\right)-\frac{d}{d \lambda_{i}}\left(\delta\left(\rho_{i j}\right) \frac{\partial F_{i j k}}{\partial \dot{z}_{i \mu}}\right)\right)\right. \\
& +\delta\left(\rho_{j k}\right)\left(\frac{\partial}{\partial z_{i \mu}}\left(\delta\left(\rho_{k i}\right) F_{j k i}\right)-\frac{d}{d \lambda_{i}}\left(\delta\left(\rho_{k i}\right) \frac{\partial F_{j k i}}{\partial \dot{z}_{i \mu}}\right)\right) \\
& \left.+\frac{\partial}{\partial z_{i \mu}}\left(\delta\left(\rho_{k i}\right) \delta\left(\rho_{i j}\right) F_{k i j}\right)-\frac{d}{d \lambda_{i}}\left(\delta\left(\rho_{k i}\right) \delta\left(\rho_{i j}\right) \frac{\partial F_{k i j}}{\partial \dot{z}_{i \mu}}\right)\right)+\ldots \\
& =0 .
\end{aligned}
$$

Integrating by parts and taking into account that:

$$
\frac{d}{d \lambda_{i}}\left(\delta\left(\rho_{i j}\right)\right)=\frac{\left(\frac{d \rho_{i j}}{d \lambda_{i}}\right)}{\left(\frac{d \rho_{i j}}{d \lambda_{j}}\right)} \frac{d}{d \lambda_{j}}\left(\delta\left(\rho_{i j}\right)\right)=\frac{\gamma_{i j}}{\gamma_{j i}} \frac{d}{d \lambda_{j}}\left(\delta\left(\rho_{i j}\right)\right),
$$

we can write the equations of motion (8) in the form:

$$
\begin{aligned}
\ddot{z}_{i}^{\mu} & +\frac{G}{c^{2}} \sum_{j \neq i} m_{j} \int d \lambda_{j} \delta\left(\rho_{i j}\right)\left(A_{i j}^{\mu}+B_{i j}^{\mu \nu} \ddot{z}_{i \nu}+C_{i j}^{\mu \nu} \ddot{z}_{j \nu}\right) \\
+ & \frac{G^{2}}{c^{4}} \sum_{j \neq i} \sum_{k \neq i, j} m_{j} m_{k} \iint d \lambda_{j} d \lambda_{k} \delta\left(\rho_{i j}\right) \delta\left(\rho_{j k}\right)\left(A_{i j k}^{\mu}+B_{i j k}^{\mu \nu} \ddot{z}_{i \nu}+C_{i j k}^{\mu \nu} \ddot{z}_{j \nu}+D_{i j k}^{\mu \nu} \ddot{z}_{k \nu}\right) \\
+ & \frac{G^{2}}{2 c^{4}} \sum_{j \neq i} \sum_{k \neq i, j} m_{j} m_{k} \iint d \lambda_{j} d \lambda_{k} \delta\left(\rho_{j i}\right) \delta\left(\rho_{i k}\right)\left(\tilde{A}_{j i k}^{\mu}+\tilde{B}_{j i k}^{\mu \nu} \ddot{z}_{i \nu}+\tilde{C}_{j i k}^{\mu \nu} \ddot{z}_{j \nu}+\tilde{D}_{j i k}^{\mu \nu} \ddot{z}_{k \nu}\right)+\ldots \\
& =0
\end{aligned}
$$

where

$$
\begin{gathered}
A_{i j}^{\mu}=\frac{\partial F_{i j}}{\partial z_{i \mu}}-\frac{\partial^{2} F_{i j}}{\partial z_{i}^{\eta} \partial \dot{z}_{i \mu}} \dot{z}_{i}^{\eta}+\frac{\zeta_{j}}{\gamma_{j i}^{2}}\left(\left(z_{i}^{\mu}-z_{j}^{\mu}\right) F_{i j}+\gamma_{i j} \frac{\partial F_{i j}}{\partial \dot{z}_{i \mu}}\right) \\
+\frac{1}{\gamma_{j i}}\left(-\dot{z}_{j}^{\mu} F_{i j}+\left(z_{i}^{\mu}-z_{j}^{\mu}\right) \frac{\partial F_{i j}}{\partial z_{j}^{\eta}} \dot{z}_{j}^{\eta}+\xi_{i j} \frac{\partial F_{i j}}{\partial \dot{z}_{i \mu}}+\gamma_{i j} \frac{\partial^{2} F_{i j}}{\partial z_{j}^{\eta} \partial \dot{z}_{i \mu}} \dot{z}_{j}^{\eta}\right) \\
B_{i j}^{\mu \nu}=-\frac{\partial^{2} F_{i j}}{\partial \dot{z}_{i \mu} \partial \dot{z}_{i \nu}}, \\
C_{i j}^{\mu \nu}=\frac{\left(z_{i}^{\mu}-z_{j}^{\mu}\right)}{\gamma_{j i}}\left(\frac{\partial F_{i j}}{\partial \dot{z}_{j \nu}}-\frac{\left(z_{i}^{\nu}-z_{j}^{\nu}\right)}{\gamma_{j i}} F_{i j}\right)+\frac{\gamma_{i j}}{\gamma_{j i}}\left(\frac{\partial^{2} F_{i j}}{\partial \dot{z}_{i \mu} \partial \dot{z}_{j \nu}}-\frac{\left(z_{i}^{\nu}-z_{j}^{\nu}\right)}{\gamma_{j i}} \frac{\partial F_{i j}}{\partial \dot{z}_{i \mu}}\right),
\end{gathered}
$$




$$
\begin{aligned}
& A_{i j k}^{\mu}=\frac{\partial F_{i j k}}{\partial z_{i \mu}}-\frac{\partial^{2} F_{i j k}}{\partial z_{i}^{\eta} \partial \dot{z}_{i \mu}} \dot{z}_{i}^{\eta}+\frac{\zeta_{j}}{\gamma_{j i}^{2}}\left(\left(z_{i}^{\mu}-z_{j}^{\mu}\right) F_{i j k}+\gamma_{i j} \frac{\partial F_{i j k}}{\partial \dot{z}_{i \mu}}\right) \\
& +\frac{1}{\gamma_{j i}}\left(-\dot{z}_{j}^{\mu} F_{i j k}+\left(z_{i}^{\mu}-z_{j}^{\mu}\right) \frac{\partial F_{i j k}}{\partial z_{j}^{\eta}} \dot{z}_{j}^{\eta}+\xi_{i j} \frac{\partial F_{i j k}}{\partial \dot{z}_{i \mu}}+\gamma_{i j} \frac{\partial^{2} F_{i j k}}{\partial z_{j}^{\eta} \partial \dot{z}_{i \mu}} \dot{z}_{j}^{\eta}\right) \\
& -\frac{1}{\gamma_{k j} \gamma_{j i}}\left(\xi_{j k}+\frac{\gamma_{j k}}{\gamma_{k j}} \zeta_{k}\right)\left(\left(z_{i}^{\mu}-z_{j}^{\mu}\right) F_{i j k}+\gamma_{i j} \frac{\partial F_{i j k}}{\partial \dot{z}_{i \mu}}\right) \\
& -\frac{\gamma_{j k}}{\gamma_{k j} \gamma_{j i}}\left(\left(z_{i}^{\mu}-z_{j}^{\mu}\right) \frac{\partial F_{i j k}}{\partial z_{k}^{\eta}} \dot{z}_{k}^{\eta}+\gamma_{i j} \frac{\partial^{2} F_{i j k}}{\partial z_{k}^{\eta} \partial \dot{z}_{i \mu}} \dot{z}_{k}^{\eta}\right) \\
& B_{i j k}^{\mu \nu}=-\frac{\partial^{2} F_{i j k}}{\partial \dot{z}_{i \mu} \partial \dot{z}_{i \nu}} \\
& C_{i j k}^{\mu \nu}=\frac{\left(z_{i}^{\mu}-z_{j}^{\mu}\right)}{\gamma_{j i}}\left(\frac{\partial F_{i j k}}{\partial \dot{z}_{j \nu}}-\frac{\left(z_{i}^{\nu}-z_{j}^{\nu}\right)}{\gamma_{j i}} F_{i j k}\right)+\frac{\gamma_{i j}}{\gamma_{j i}}\left(\frac{\partial^{2} F_{i j k}}{\partial \dot{z}_{i \mu} \partial \dot{z}_{j \nu}}-\frac{\left(z_{i}^{\nu}-z_{j}^{\nu}\right)}{\gamma_{j i}} \frac{\partial F_{i j k}}{\partial \dot{z}_{i \mu}}\right), \\
& D_{i j k}^{\mu \nu}=\frac{\gamma_{j k}\left(z_{j}^{\nu}-z_{k}^{\nu}\right)}{\gamma_{k j}^{2}}\left(\frac{\left(z_{i}^{\mu}-z_{j}^{\mu}\right)}{\gamma_{j i}} F_{i j k}+\frac{\gamma_{i j}}{\gamma_{j i}} \frac{\partial F_{i j k}}{\partial \dot{z}_{i \mu}}\right)-\frac{\gamma_{j k}}{\gamma_{k j}}\left(\frac{\left(z_{i}^{\mu}-z_{j}^{\mu}\right)}{\gamma_{j i}} \frac{\partial F_{i j k}}{\partial \dot{z}_{k \nu}}+\frac{\gamma_{i j}}{\gamma_{j i}} \frac{\partial^{2} F_{i j k}}{\partial \dot{z}_{i \mu} \partial \dot{z}_{k \nu}}\right) \\
& \tilde{A}_{j i k}^{\mu}=\frac{\partial F_{j i k}}{\partial z_{i \mu}}-\frac{\partial^{2} F_{j i k}}{\partial z_{i}^{\eta} \partial \dot{z}_{i \mu}} \dot{z}_{i}^{\eta}+\frac{\zeta_{j}}{\gamma_{j i}^{2}}\left(\left(z_{i}^{\mu}-z_{j}^{\mu}\right) F_{j i k}+\gamma_{i j} \frac{\partial F_{j i k}}{\partial \dot{z}_{i \mu}}\right)+\frac{\zeta_{k}}{\gamma_{k i}^{2}}\left(\left(z_{i}^{\mu}-z_{k}^{\mu}\right) F_{j i k}+\gamma_{i k} \frac{\partial F_{j i k}}{\partial \dot{z}_{i \mu}}\right) \\
& +\frac{1}{\gamma_{j i}}\left(-\dot{z}_{j}^{\mu} F_{j i k}+\left(z_{i}^{\mu}-z_{j}^{\mu}\right) \frac{\partial F_{j i k}}{\partial z_{j}^{\eta}} \dot{z}_{j}^{\eta}+\xi_{i j} \frac{\partial F_{j i k}}{\partial \dot{z}_{i \mu}}+\gamma_{i j} \frac{\partial^{2} F_{j i k}}{\partial z_{j}^{\eta} \partial \dot{z}_{i \mu}} \dot{z}_{j}^{\eta}\right) \\
& +\frac{1}{\gamma_{k i}}\left(-\dot{z}_{k}^{\mu} F_{j i k}+\left(z_{i}^{\mu}-z_{k}^{\mu}\right) \frac{\partial F_{j i k}}{\partial z_{k}^{\eta}} \dot{z}_{k}^{\eta}+\xi_{i k} \frac{\partial F_{j i k}}{\partial \dot{z}_{i \mu}}+\gamma_{i k} \frac{\partial^{2} F_{j i k}}{\partial z_{k}^{\eta} \partial \dot{z}_{i \mu}} \dot{z}_{k}^{\eta}\right) \\
& \tilde{B}_{j i k}^{\mu \nu}=-\frac{\partial^{2} F_{j i k}}{\partial \dot{z}_{i \mu} \partial \dot{z}_{i \nu}} \\
& \tilde{C}_{j i k}^{\mu \nu}=\frac{\left(z_{i}^{\mu}-z_{j}^{\mu}\right)}{\gamma_{j i}}\left(\frac{\partial F_{j i k}}{\partial \dot{z}_{j \nu}}-\frac{\left(z_{i}^{\nu}-z_{j}^{\nu}\right)}{\gamma_{j i}} F_{j i k}\right)+\frac{\gamma_{i j}}{\gamma_{j i}}\left(\frac{\partial^{2} F_{j i k}}{\partial \dot{z}_{i \mu} \partial \dot{z}_{j \nu}}-\frac{\left(z_{i}^{\nu}-z_{j}^{\nu}\right)}{\gamma_{j i}} \frac{\partial F_{j i k}}{\partial \dot{z}_{i \mu}}\right), \\
& \tilde{D}_{j i k}^{\mu \nu}=-\frac{\left(z_{i}^{\nu}-z_{k}^{\nu}\right)}{\gamma_{k i}^{2}}\left(\left(z_{i}^{\mu}-z_{k}^{\mu}\right) F_{j i k}+\gamma_{i k} \frac{\partial F_{j i k}}{\partial \dot{z}_{i \mu}}\right)+\frac{1}{\gamma_{k i}}\left(\left(z_{i}^{\mu}-z_{k}^{\mu}\right) \frac{\partial F_{j i k}}{\partial \dot{z}_{k \nu}}+\gamma_{i k} \frac{\partial^{2} F_{j i k}}{\partial \dot{z}_{i \mu} \partial \dot{z}_{k \nu}}\right) .
\end{aligned}
$$

Multiplying (8) by $\dot{z}_{i \mu}$ (and performing the summation over $\mu$ ), we find that the solutions of the equations of motion (8) must satisfy the following $N$ conditions $(i=1,2, \ldots, N)$ : 


$$
\begin{aligned}
& \frac{d}{d \lambda_{i}}\left(\zeta_{i}+\frac{2 G}{c^{2}} \sum_{j \neq i} m_{j} \int d \lambda_{j} \delta\left(\rho_{i j}\right)\left(F_{i j}-\dot{z}_{i}^{\mu} \frac{\partial F_{i j}}{\partial \dot{z}_{i}^{\mu}}\right)\right. \\
& +\frac{G^{2}}{c^{4}} \sum_{j \neq i} \sum_{k \neq i, j} m_{j} m_{k} \iint d \lambda_{j} d \lambda_{k}\left(\delta\left(\rho_{i j}\right) \delta\left(\rho_{j k}\right)\left(F_{i j k}-\dot{z}_{i}^{\mu} \frac{\partial F_{i j k}}{\partial \dot{z}_{i}^{\mu}}\right)\right. \\
& \left.\left.+\delta\left(\rho_{j k}\right) \delta\left(\rho_{k i}\right)\left(F_{j k i}-\dot{z}_{i}^{\mu} \frac{\partial F_{j k i}}{\partial \dot{z}_{i}^{\mu}}\right)+\delta\left(\rho_{k i}\right) \delta\left(\rho_{i j}\right)\left(F_{k i j}-\dot{z}_{i}^{\mu} \frac{\partial F_{k i j}}{\partial \dot{z}_{i}^{\mu}}\right)\right)+\ldots\right) \\
& =0 .
\end{aligned}
$$

Let us assume that $F_{i_{1} \ldots i_{k}}(k=2, \ldots, N)$ are homogeneous functions of degree two in $\dot{z}_{i_{1}}$, $\ldots, \dot{z}_{i_{k}}$, i.e., we assume that they satisfy the following conditions:

$$
\dot{z}_{i_{1}}^{\mu} \frac{\partial F_{i_{1} \ldots i_{k}}}{\partial \dot{z}_{i_{1}}^{\mu}}=\ldots=\dot{z}_{i_{k}}^{\mu} \frac{\partial F_{i_{1} \ldots i_{k}}}{\partial \dot{z}_{i_{k}}^{\mu}}=2 F_{i_{1} \ldots i_{k}}
$$

The conditions (22) combined with (23) guarantee that, for the solutions of the equations of motion, the expressions:

$$
\begin{aligned}
\zeta_{i} & -\frac{2 G}{c^{2}} \sum_{j \neq i} m_{j} \int d \lambda_{j} \delta\left(\rho_{i j}\right) F_{i j} \\
& -\frac{G^{2}}{c^{4}} \sum_{j \neq i} \sum_{k \neq i, j} m_{j} m_{k} \iint d \lambda_{j} d \lambda_{k}\left(\delta\left(\rho_{i j}\right) \delta\left(\rho_{j k}\right) F_{i j k}+\delta\left(\rho_{j k}\right) \delta\left(\rho_{k i}\right) F_{j k i}+\delta\left(\rho_{k i}\right) \delta\left(\rho_{i j}\right) F_{k i j}\right)+\ldots \\
& =c_{i},
\end{aligned}
$$

are constants (which by simple scaling can be made equal to 1 ):

$$
\begin{aligned}
\zeta_{i} & -\frac{2 G}{c^{2}} \sum_{j \neq i} m_{j} \int d \lambda_{j} \delta\left(\rho_{i j}\right) F_{i j} \\
& -\frac{G^{2}}{c^{4}} \sum_{j \neq i} \sum_{k \neq i, j} m_{j} m_{k} \iint d \lambda_{j} d \lambda_{k}\left(\delta\left(\rho_{i j}\right) \delta\left(\rho_{j k}\right) F_{i j k}+\delta\left(\rho_{j k}\right) \delta\left(\rho_{k i}\right) F_{j k i}+\delta\left(\rho_{k i}\right) \delta\left(\rho_{i j}\right) F_{k i j}\right)+\ldots \\
& =1 .
\end{aligned}
$$

From (23) it immediately follows that:

$$
F_{i_{1} \ldots i_{k}}=\frac{1}{2} \frac{\partial^{2} F_{i_{1} \ldots i_{k}}}{\partial \dot{z}_{i_{1}}^{\mu} \partial \dot{z}_{i_{1}}^{\nu}} \dot{z}_{i_{1}}^{\mu} \dot{z}_{i_{1}}^{\nu}=\ldots=\frac{1}{2} \frac{\partial^{2} F_{i_{1} \ldots i_{k}}}{\partial \dot{z}_{i_{k}}^{\mu} \partial \dot{z}_{i_{k}}^{\nu}} \dot{z}_{i_{k}}^{\mu} \dot{z}_{i_{k}}^{\nu}
$$

Using (26) we immediately see that the action for particle $i$ (7) can be rewritten in a compact form as:

$$
S_{i}=-m_{i} c \int d \lambda_{i} g_{\mu \nu}^{(i)} \dot{z}_{i}^{\mu} \dot{z}_{i}^{\nu}
$$

where, 


$$
\begin{aligned}
g_{\mu \nu}^{(i)}= & \eta_{\mu \nu}-\frac{G}{c^{2}} \sum_{j \neq i} m_{j} \int d \lambda_{j} \delta\left(\rho_{i j}\right) \frac{\partial^{2} F_{i j}}{\partial \dot{z}_{i}^{\mu} \partial \dot{z}_{i}^{\nu}} \\
& -\frac{G^{2}}{2 c^{4}} \sum_{j \neq i} \sum_{k \neq i, j} m_{j} m_{k} \iint d \lambda_{j} d \lambda_{k}\left(\delta\left(\rho_{i j}\right) \delta\left(\rho_{j k}\right) \frac{\partial^{2} F_{i j k}}{\partial \dot{z}_{i}^{\mu} \partial \dot{z}_{i}^{\nu}}+\delta\left(\rho_{j k}\right) \delta\left(\rho_{k i}\right) \frac{\partial^{2} F_{j k i}}{\partial \dot{z}_{i}^{\mu} \partial \dot{z}_{i}^{\nu}}\right. \\
& \left.+\delta\left(\rho_{k i}\right) \delta\left(\rho_{i j}\right) \frac{\partial^{2} F_{k i j}}{\partial \dot{z}_{i}^{\mu} \partial \dot{z}_{i}^{\nu}}\right)+\ldots
\end{aligned}
$$

From (28, 23, 26) it follows that:

$$
\begin{gathered}
\frac{\partial g_{\alpha \beta}^{(i)}}{\partial \dot{z}_{i}^{\mu}} \dot{z}_{i}^{\alpha}=0, \\
\frac{\partial^{2} g_{\alpha \beta}^{(i)}}{\partial \dot{z}_{i}^{\mu} \partial \dot{z}_{i}^{\nu}} \dot{z}_{i}^{\alpha} \dot{z}_{i}^{\beta}=0, \\
\frac{\partial^{2} g_{\alpha \beta}^{(i)}}{\partial \dot{z}_{i}^{\mu} \partial z_{i}^{\nu}} \dot{z}_{i}^{\alpha}=0 .
\end{gathered}
$$
form:

Using (28, 29, 30, 31) the equations of motion (8) can also be written in a more compact

$$
g_{\mu \nu}^{(i)} \ddot{z}_{i}^{\nu}+\frac{1}{2}\left(\frac{\partial g_{\mu \alpha}^{(i)}}{\partial z_{i}^{\beta}}+\frac{\partial g_{\mu \beta}^{(i)}}{\partial z_{i}^{\alpha}}-\frac{\partial g_{\alpha \beta}^{(i)}}{\partial z_{i}^{\mu}}\right) \dot{z}_{i}^{\alpha} \dot{z}_{i}^{\beta}=0
$$

From (10, 22, 28), we find that the conditions (22) can be simply expressed as:

$$
\frac{d}{d \lambda_{i}}\left(g_{\mu \nu}^{(i)} \dot{z}_{i}^{\mu} \dot{z}_{i}^{\nu}\right)=0
$$

From (25, 26, 28) it follows that for the solutions of the equations of motion:

$$
d \lambda_{i}^{2}=g_{\mu \nu}^{(i)} d z_{i}^{\mu} d z_{i}^{\nu}
$$

Notice that $g_{\mu \nu}^{(i)}$ is not a field. It depends not only on $z_{i}, z_{j}$ and $\dot{z}_{j}(j \neq i)$, but also on $\dot{z}_{i}$ !

The main task in our formulation is to determine the functions $F_{i j}, F_{i j k}$, etc, in (6) ), and to verify that the predictions of the theory are in agreement with observations.

Test particles and the formulation of the action-at-a-distance model as a field theory

Let us assume that in the limit $m_{i} \rightarrow 0$ the tensor $g_{\mu \nu}^{(i)}$ does not depend on $\dot{z}_{i}$. Only in this limit, in which $m_{i}$ is a test particle, we may have a field interpretation for the metric tensor $g_{\mu \nu}^{(i)}$.

Let us consider a system of $N+1$ point particles, one of them being a test particle of mass $m$ and the other $N$ particles having masses $m_{i}(i=1, \ldots, N)$. Let $z(\lambda)$ be the worldline of the test particle. From (27) we see that we can write the action for the test particle as follows:

$$
S=-m c \int d \lambda g_{\mu \nu} \dot{z}^{\mu} \dot{z}^{\nu}
$$


In (35), the metric tensor $g_{\mu \nu}$ depends on $z$ (and on $z_{i}$ and $\dot{z}_{i}(i=1, \ldots, N)$ ), but does not depend on $\dot{z}$. It can be given a field interpretation, if one desires to do so[54].

From (32) we see that for a test particle the equations of motion are:

$$
g_{\mu \nu} \ddot{z}^{\nu}+\frac{1}{2}\left(\frac{\partial g_{\mu \alpha}}{\partial z^{\beta}}+\frac{\partial g_{\mu \beta}}{\partial z^{\alpha}}-\frac{\partial g_{\alpha \beta}}{\partial z^{\mu}}\right) \dot{z}^{\alpha} \dot{z}^{\beta}=0
$$

Assuming that the matrix $g_{\mu \nu}$ is invertible, these are, of course, the well known equations for geodesics.

It may be possible to impose conditions on the functions $F_{i j}, F_{i j k}$, etc, in (6) if, for example, one demands that the metric tensor $g_{\mu \nu}$ (which is associated with a test particle) obeys Einstein's field equations. Of course, there is no guarantee that this can be done at all orders in the Post-Minkowskian expansion, either due to the mathematical complexity of the equations or to the possibility that Einstein's theory of General Relativity may not exactly admit a dual (action-at-a-distance) formulation, or at least not one described by an action of the form (6). At the first Post-Minkowskian order it is known that $F_{i j}=2 \xi_{i j}^{2}-\zeta_{i j}^{2}$ [50]- [53].

A possible expression for $F_{i j}$

Let us assume that the functions $F_{i j}$ can be expressed as follows:

$$
F_{i j}=\alpha\left(\epsilon_{i j}, \epsilon_{j i}\right) \xi_{i j}^{2}+\beta\left(\epsilon_{i j}, \epsilon_{j i}\right) \zeta_{i j}^{2},
$$

where,

$$
\epsilon_{i j}=\frac{G m_{i}}{2 c^{2}\left|\eta_{i j}\right|}
$$

and 49$]$ :

$$
\begin{gathered}
\zeta_{i j}=\sqrt{\zeta_{i} \zeta_{j}}, \\
\eta_{i j}=\frac{\gamma_{i j}}{\sqrt{\zeta_{i}}} .
\end{gathered}
$$

We assume that the functions $\alpha$ and $\beta$ are symmetric:

$$
\begin{gathered}
\alpha\left(\epsilon_{i j}, \epsilon_{j i}\right)=\alpha\left(\epsilon_{j i}, \epsilon_{i j}\right), \\
\beta\left(\epsilon_{i j}, \epsilon_{j i}\right)=\beta\left(\epsilon_{j i}, \epsilon_{i j}\right) .
\end{gathered}
$$

The one-body problem

Let us consider the case of a test particle interacting with a particle of mass $M$. This is the case $N=1$ (the one-body problem). The motion of the mass $M$ is not affected by the presence of the test particle. The mass $M$ moves with constant velocity in any inertial reference frame.

Let us, for simplicity, consider the inertial frame in which the mass $M$ is at rest and positioned at the origin of the coordinate system. In this frame of reference the world line of the test particle is described by the four-vector $z^{\mu}=(c t, \vec{r})$. From (28) and (37) we find the components of the metric tensor $g_{\mu \nu}$ in this reference frame to be as follows:

$$
\begin{gathered}
g_{00}=1-\frac{2 G M(\alpha(0, \epsilon)+\beta(0, \epsilon))}{c^{2} r}, \\
g_{0 i}=0,
\end{gathered}
$$




$$
g_{i j}=-\delta_{i j}\left(1-\frac{2 G M \beta(0, \epsilon)}{c^{2} r}\right) .
$$

In (43- - 45):

$$
\epsilon=\frac{G M}{2 c^{2} r}
$$

If we choose the functions $\alpha$ and $\beta$ as follows:

$$
\begin{gathered}
\alpha(0, \epsilon)=\frac{(1+\epsilon)^{4}-\frac{(1-\epsilon)^{2}}{(1+\epsilon)^{2}}}{4 \epsilon}, \\
\beta(0, \epsilon)=\frac{1-(1+\epsilon)^{4}}{4 \epsilon},
\end{gathered}
$$

one can easily check that the metric (43-45), with $\alpha$ and $\beta$ given by (47],48), coincides with the well known Schwarzschild metric of GR in isotropic form 55 .

Since $\alpha, \beta$ and $\epsilon_{j i}$ are Poincaré invariants, we can write the functional relations:

$$
\begin{gathered}
\alpha\left(0, \epsilon_{j i}\right)=\frac{\left(1+\epsilon_{j i}\right)^{4}-\frac{\left(1-\epsilon_{j i}\right)^{2}}{\left(1+\epsilon_{j i}\right)^{2}}}{4 \epsilon_{j i}}, \\
\beta\left(0, \epsilon_{j i}\right)=\frac{1-\left(1+\epsilon_{j i}\right)^{4}}{4 \epsilon_{j i}} .
\end{gathered}
$$

At the second Post-Minkowskian order (up to terms proportional to $G^{2}$ in the metric), we can write:

$$
\begin{aligned}
& \alpha\left(0, \epsilon_{j i}\right) \approx \alpha_{0}+\alpha_{1} \epsilon_{j i}, \\
& \beta\left(0, \epsilon_{j i}\right) \approx \beta_{0}+\beta_{1} \epsilon_{j i},
\end{aligned}
$$

where $\alpha_{0}, \beta_{0}, \alpha_{1}$ and $\beta_{1}$ are constants.

The values of these constants can easily be determined by expanding (49) and (50). We find:

$$
\begin{aligned}
\alpha_{0} & =2, \\
\beta_{0} & =-1, \\
\alpha_{1} & =-\frac{1}{2}, \\
\beta_{1} & =-\frac{3}{2} .
\end{aligned}
$$

The second Post-Minkowskian approximation

Let us now consider the gravitational $N$-body problem described by the action (6) . Assume that $F_{i j}$ are given by (37, 41,42). At the second Post-Minkowskian (2PM) order the functions $\alpha$ and $\beta$ will be given by the expressions:

$$
\begin{gathered}
\alpha\left(\epsilon_{i j}, \epsilon_{j i}\right) \approx \alpha_{0}+\alpha_{1}\left(\epsilon_{i j}+\epsilon_{j i}\right), \\
\beta\left(\epsilon_{i j}, \epsilon_{j i}\right) \approx \beta_{0}+\beta_{1}\left(\epsilon_{i j}+\epsilon_{j i}\right) .
\end{gathered}
$$


Let us consider the case where the functions $F_{i j k}$ can be written as:

$$
\begin{aligned}
F_{i j k}= & a\left(\epsilon_{i j}, \epsilon_{j i}, \epsilon_{k i}, \epsilon_{i k}, \epsilon_{j k}, \epsilon_{k j}\right) \xi_{i j} \xi_{j k} \xi_{k i} \\
& +b\left(\epsilon_{i j}, \epsilon_{j i}, \epsilon_{k i}, \epsilon_{i k}, \epsilon_{j k}, \epsilon_{k j}\right) \zeta_{i} \zeta_{j} \zeta_{k} \\
& +c\left(\epsilon_{i j}, \epsilon_{j i}, \epsilon_{k i}, \epsilon_{i k}, \epsilon_{j k}, \epsilon_{k j}\right) \xi_{k i}^{2} \zeta_{j}
\end{aligned}
$$

We assume that the functions $a, b$ and $c$ are symmetric in the indexes $(i k)$ :

$$
\begin{aligned}
& a\left(\epsilon_{k j}, \epsilon_{j k}, \epsilon_{i k}, \epsilon_{k i}, \epsilon_{j i}, \epsilon_{i j}\right)=a\left(\epsilon_{i j}, \epsilon_{j i}, \epsilon_{k i}, \epsilon_{i k}, \epsilon_{j k}, \epsilon_{k j}\right), \\
& b\left(\epsilon_{k j}, \epsilon_{j k}, \epsilon_{i k}, \epsilon_{k i}, \epsilon_{j i}, \epsilon_{i j}\right)=b\left(\epsilon_{i j}, \epsilon_{j i}, \epsilon_{k i}, \epsilon_{i k}, \epsilon_{j k}, \epsilon_{k j}\right), \\
& c\left(\epsilon_{k j}, \epsilon_{j k}, \epsilon_{i k}, \epsilon_{k i}, \epsilon_{j i}, \epsilon_{i j}\right)=c\left(\epsilon_{i j}, \epsilon_{j i}, \epsilon_{k i}, \epsilon_{i k}, \epsilon_{j k}, \epsilon_{k j}\right) .
\end{aligned}
$$

At $2 \mathrm{PM}$ we have:

$$
\begin{aligned}
& a\left(\epsilon_{i j}, \epsilon_{j i}, \epsilon_{k i}, \epsilon_{i k}, \epsilon_{j k}, \epsilon_{k j}\right) \approx a_{0}, \\
& b\left(\epsilon_{i j}, \epsilon_{j i}, \epsilon_{k i}, \epsilon_{i k}, \epsilon_{j k}, \epsilon_{k j}\right) \approx b_{0}, \\
& c\left(\epsilon_{i j}, \epsilon_{j i}, \epsilon_{k i}, \epsilon_{i k}, \epsilon_{j k}, \epsilon_{k j}\right) \approx c_{0},
\end{aligned}
$$

where $a_{0}, b_{0}$ and $c_{0}$ are constants.

Therefore, at the second Post-Minkowskian order we can write the action, for a system of $N$ particles interacting gravitationally, as follows:

$$
\begin{aligned}
S= & -\sum_{i} m_{i} c \int d \lambda_{i} \zeta_{i}+\sum_{i} \sum_{j \neq i} \frac{G m_{i} m_{j}}{c} \iint d \lambda_{i} d \lambda_{j} \delta\left(\rho_{i j}\right) F_{i j} \\
& +\sum_{i} \sum_{j \neq i} \sum_{k \neq i, j} \frac{G^{2} m_{i} m_{j} m_{k}}{c^{3}} \iiint d \lambda_{i} d \lambda_{j} d \lambda_{k} \delta\left(\rho_{i j}\right) \delta\left(\rho_{j k}\right) F_{i j k},
\end{aligned}
$$

where,

$$
\begin{gathered}
F_{i j}=\left(\alpha_{0}+\alpha_{1}\left(\epsilon_{i j}+\epsilon_{j i}\right)\right) \xi_{i j}^{2}+\left(\beta_{0}+\beta_{1}\left(\epsilon_{i j}+\epsilon_{j i}\right)\right) \zeta_{i j}^{2} \\
F_{i j k}=a_{0} \xi_{i j} \xi_{j k} \xi_{k i}+b_{0} \zeta_{i} \zeta_{j} \zeta_{k}+c_{0} \xi_{k i}^{2} \zeta_{j}
\end{gathered}
$$

At the second Post-Minkowskian approximation there is no need to consider the functions $F_{i_{1} \ldots i_{k}}$ for $k>3$ since the terms associated with these functions in the action (6) give contributions only at the $(k-1)$-Post-Minkowskian order.

At the second Post-Minkowskian order (2PM) the equations of motion are:

$$
\begin{aligned}
\ddot{z}_{i}^{\mu} & +\frac{G}{c^{2}} \sum_{j \neq i} m_{j} \int d \lambda_{j} \delta\left(\rho_{i j}\right)\left(A_{i j}^{(0) \mu}+A_{i j}^{(1) \mu}+B_{i j}^{(0) \mu \nu} \ddot{z}_{i \nu}+C_{i j}^{(0) \mu \nu} \ddot{z}_{j \nu}\right) \\
& +\frac{G^{2}}{c^{4}} \sum_{j \neq i} \sum_{k \neq i, j} m_{j} m_{k} \iint d \lambda_{j} d \lambda_{k} \delta\left(\rho_{i j}\right) \delta\left(\rho_{j k}\right) A_{i j k}^{(0) \mu} \\
& +\frac{G^{2}}{2 c^{4}} \sum_{j \neq i} \sum_{k \neq i, j} m_{j} m_{k} \iint d \lambda_{j} d \lambda_{k} \delta\left(\rho_{j i}\right) \delta\left(\rho_{i k}\right) \tilde{A}_{j i k}^{(0) \mu} \\
& =0
\end{aligned}
$$


Substituting (67, 68) into (11- 13) and (14, 18) we find (in this approximation):

$$
\begin{aligned}
& A_{i j}^{(0) \mu}=\frac{\left(z_{i}^{\mu}-z_{j}^{\mu}\right)}{\eta_{j i}^{2}}\left(\alpha_{0} \xi_{i j}^{2}+\beta_{0} \zeta_{i j}^{2}\right)+\frac{2 \dot{z}_{i}^{\mu}}{\zeta_{i}^{\frac{1}{2}} \eta_{j i}} \zeta_{i j}^{2}\left(\frac{\xi_{i j}}{\zeta_{i j}}+\frac{\eta_{i j}}{\eta_{j i}}\right) \beta_{0} \\
& +\frac{\dot{z}_{j}^{\mu}}{\zeta_{j}^{\frac{1}{2}} \eta_{j i}}\left[\alpha_{0}\left(\xi_{i j}^{2}+2 \xi_{i j} \zeta_{i j} \frac{\eta_{i j}}{\eta_{j i}}\right)-\beta_{0} \zeta_{i j}^{2}\right] \text {, } \\
& A_{i j}^{(1) \mu}=\frac{2\left(z_{i}^{\mu}-z_{j}^{\mu}\right)}{\eta_{j i}^{2}}\left(\epsilon_{j i}-\epsilon_{i j}\left(1+\frac{\xi_{i j} \eta_{i j}}{\zeta_{i j} \eta_{j i}}-\frac{\eta_{i j}^{2}}{\eta_{j i}^{2}}\right)\right)\left(\alpha_{1} \xi_{i j}^{2}+\beta_{1} \zeta_{i j}^{2}\right) \\
& +\frac{\dot{z}_{i}^{\mu}}{\zeta_{i}^{\frac{1}{2}} \eta_{j i}}\left[4 \epsilon_{j i} \zeta_{i j}^{2}\left(\frac{\xi_{i j}}{\zeta_{i j}}+\frac{\eta_{i j}}{\eta_{j i}}\right) \beta_{1}-\epsilon_{i j}\left(1-\frac{\eta_{i j}^{2}}{\eta_{j i}^{2}}\right)\left(\alpha_{1} \xi_{i j}^{2}+3 \beta_{1} \zeta_{i j}^{2}\right)\right] \\
& +\frac{2 \dot{z}_{j}^{\mu}}{\zeta_{j}^{\frac{1}{2}} \eta_{j i}}\left[\epsilon_{j i}\left(\alpha_{1}\left(2 \xi_{i j} \zeta_{i j} \frac{\eta_{i j}}{\eta_{j i}}+\xi_{i j}^{2}\right)-\beta_{1} \zeta_{i j}^{2}\right)\right. \\
& \left.-\epsilon_{i j}\left(\alpha_{1}\left(\xi_{i j}^{2}+\xi_{i j} \zeta_{i j}\left(\frac{\eta_{j i}}{\eta_{i j}}-\frac{\eta_{i j}}{\eta_{j i}}\right)\right)+\beta_{1} \zeta_{i j}^{2}\right)\right] \text {, } \\
& B_{i j}^{(0) \mu \nu}=-2 \alpha_{0} \dot{z}_{j}^{\mu} \dot{z}_{j}^{\nu}-2 \beta_{0} \zeta_{j} \eta^{\mu \nu} \\
& C_{i j}^{(0) \mu \nu}=-\frac{\left(z_{i}^{\mu}-z_{j}^{\mu}\right)\left(z_{i}^{\nu}-z_{j}^{\nu}\right)}{\zeta_{j} \eta_{j i}^{2}}\left(\alpha_{0} \xi_{i j}^{2}+\beta_{0} \zeta_{i j}^{2}\right)+\frac{2\left(z_{i}^{\mu}-z_{j}^{\mu}\right)}{\zeta_{j}^{\frac{1}{2}} \eta_{j i}}\left(\alpha_{0} \xi_{i j} \dot{z}_{i}^{\nu}+\beta_{0} \zeta_{i} \dot{z}_{j}^{\nu}\right) \\
& -\frac{2\left(z_{i}^{\nu}-z_{j}^{\nu}\right) \zeta_{i}^{\frac{1}{2}} \eta_{i j}}{\zeta_{j} \eta_{j i}^{2}}\left(\alpha_{0} \xi_{i j} \dot{z}_{j}^{\mu}+\beta_{0} \zeta_{j} \dot{z}_{i}^{\mu}\right) \\
& +2 \frac{\eta_{i j} \zeta_{i}^{\frac{1}{2}}}{\eta_{j i} \zeta_{j}^{\frac{1}{2}}}\left(\alpha_{0}\left(\eta^{\mu \nu} \xi_{i j}+\dot{z}_{j}^{\mu} \dot{z}_{i}^{\nu}\right)+2 \beta_{0} \dot{z}_{i}^{\mu} \dot{z}_{j}^{\nu}\right) \\
& A_{i j k}^{(0) \mu}=\frac{\left(z_{i}^{\mu}-z_{j}^{\mu}\right)}{\gamma_{j i}}\left(\frac{\zeta_{j}}{\gamma_{j i}}-\frac{1}{\gamma_{k j}}\left(\xi_{j k}+\frac{\gamma_{j k}}{\gamma_{k j}} \zeta_{k}\right)\right)\left(a_{0} \xi_{i j} \xi_{j k} \xi_{k i}+b_{0} \zeta_{i} \zeta_{j} \zeta_{k}+c_{0} \xi_{k i}^{2} \zeta_{j}\right) \\
& +\frac{2 \dot{z}_{i}^{\mu}}{\gamma_{j i}}\left(\zeta_{j} \frac{\gamma_{i j}}{\gamma_{j i}}+\xi_{i j}-\frac{\gamma_{i j}}{\gamma_{k j}}\left(\xi_{j k}+\frac{\gamma_{j k}}{\gamma_{k j}} \zeta_{k}\right)\right) \zeta_{j} \zeta_{k} b_{0} \\
& +\frac{\dot{z}_{j}^{\mu}}{\gamma_{j i}}\left(\left(\zeta_{j} \frac{\gamma_{i j}}{\gamma_{j i}}-\frac{\gamma_{i j}}{\gamma_{k j}}\left(\xi_{j k}+\frac{\gamma_{j k}}{\gamma_{k j}} \zeta_{k}\right)\right) \xi_{j k} \xi_{k i} a_{0}-\zeta_{i} \zeta_{j} \zeta_{k} b_{0}-\xi_{k i}^{2} \zeta_{j} c_{0}\right) \\
& +\frac{\dot{z}_{k}^{\mu}}{\gamma_{j i}}\left(\zeta_{j} \frac{\gamma_{i j}}{\gamma_{j i}}+\xi_{i j}-\frac{\gamma_{i j}}{\gamma_{k j}}\left(\xi_{j k}+\frac{\gamma_{j k}}{\gamma_{k j}} \zeta_{k}\right)\right)\left(a_{0} \xi_{j k} \xi_{i j}+2 c_{0} \xi_{k i} \zeta_{j}\right)
\end{aligned}
$$




$$
\begin{aligned}
\tilde{A}_{j i k}^{(0) \mu}= & \frac{\left(z_{i}^{\mu}-z_{j}^{\mu}\right) \zeta_{j}}{\gamma_{j i}^{2}}\left(a_{0} \xi_{j i} \xi_{i k} \xi_{k j}+b_{0} \zeta_{j} \zeta_{i} \zeta_{k}+c_{0} \xi_{k j}^{2} \zeta_{i}\right) \\
& +\frac{\left(z_{i}^{\mu}-z_{k}^{\mu}\right) \zeta_{k}}{\gamma_{k i}^{2}}\left(a_{0} \xi_{j i} \xi_{i k} \xi_{k j}+b_{0} \zeta_{j} \zeta_{i} \zeta_{k}+c_{0} \xi_{k j}^{2} \zeta_{i}\right) \\
& +2 \dot{z}_{i}^{\mu}\left(\zeta_{j} \frac{\gamma_{i j}}{\gamma_{j i}^{2}}+\zeta_{k} \frac{\gamma_{i k}}{\gamma_{k i}^{2}}+\frac{\xi_{i j}}{\gamma_{j i}}+\frac{\xi_{i k}}{\gamma_{k i}}\right)\left(\zeta_{j} \zeta_{k} b_{0}+\xi_{k j}^{2} c_{0}\right) \\
& +\dot{z}_{j}^{\mu}\left(\left(\zeta_{j} \frac{\gamma_{i j}}{\gamma_{j i}^{2}}+\zeta_{k} \frac{\gamma_{i k}^{2}}{\gamma_{k i}^{2}}+\frac{\xi_{i k}}{\gamma_{k i}}\right) \xi_{k j} \xi_{i k} a_{0}-\frac{1}{\gamma_{j i}}\left(b_{0} \zeta_{j} \zeta_{i} \zeta_{k}+c_{0} \xi_{k j}^{2} \zeta_{i}\right)\right) \\
& +\dot{z}_{k}^{\mu}\left(\left(\zeta_{j} \frac{\gamma_{i j}}{\gamma_{j i}^{2}}+\zeta_{k} \frac{\gamma_{i k}}{\gamma_{k i}^{2}}+\frac{\xi_{i j}}{\gamma_{j i}}\right) \xi_{k j} \xi_{j i} a_{0}-\frac{1}{\gamma_{k i}}\left(b_{0} \zeta_{j} \zeta_{i} \zeta_{k}+c_{0} \xi_{k j}^{2} \zeta_{i}\right)\right)
\end{aligned}
$$

From (67, 68) and (28) it follows that, at the second Post-Minkowskian approximation, the metric tensor associated to the particle $i$ (with non-negligible mass $m_{i}$ ) is given by the formula:

$$
\begin{aligned}
g_{\mu \nu}^{(i)}= & \eta_{\mu \nu}-\frac{2 G}{c^{2}} \sum_{j \neq i} m_{j} \int d \lambda_{j} \delta\left(\rho_{i j}\right)\left[\alpha_{0} \dot{z}_{j \mu} \dot{z}_{j \nu}+\beta_{0} \zeta_{j} \eta_{\mu \nu}\right] \\
& -\frac{G^{2}}{c^{4}} \sum_{j \neq i} m_{j}^{2} \int d \lambda_{j} \frac{\delta\left(\rho_{i j}\right)}{\left|\eta_{j i}\right|}\left[\alpha_{1} \dot{z}_{j \mu} \dot{z}_{j \nu}+\beta_{1} \zeta_{j} \eta_{\mu \nu}\right] \\
& -\frac{G^{2} m_{i}}{c^{4}} \sum_{j \neq i} m_{j} \int d \lambda_{j} \frac{\delta\left(\rho_{i j}\right)}{\left|\eta_{i j}\right|}\left[\alpha_{1} \dot{z}_{j \mu} \dot{z}_{j \nu}+\beta_{1} \zeta_{j} \eta_{\mu \nu}+\left(\frac{\dot{z}_{i \mu}}{\zeta_{i}}+\frac{\left(z_{i \mu}-z_{j \mu}\right)}{\gamma_{i j}}\right)\left(\alpha_{1} \xi_{i j} \dot{z}_{j \nu}+\beta_{1} \zeta_{j} \dot{z}_{i \nu}\right)\right. \\
& +\left(\frac{\dot{z}_{i \nu}}{\zeta_{i}}+\frac{\left(z_{i \nu}-z_{j \nu}\right)}{\gamma_{i j}}\right)\left(\alpha_{1} \xi_{i j} \dot{z}_{j \mu}+\beta_{1} \zeta_{j} \dot{z}_{i \mu}\right)+\frac{1}{2}\left[\left(\frac{\dot{z}_{i \mu}}{\zeta_{i}}+\frac{\left(z_{i \mu}-z_{j \mu}\right)}{\gamma_{i j}}\right)\left(\frac{\dot{z}_{i \nu}}{\zeta_{i}}+\frac{\left(z_{i \nu}-z_{j \nu}\right)}{\gamma_{i j}}\right)\right. \\
& \left.\left.+\frac{1}{\zeta_{i}}\left(\eta_{\mu \nu}-\frac{2 \dot{z}_{i \mu} \dot{z}_{i \nu}}{\zeta_{i}}+\frac{\left(z_{i \mu}-z_{j \mu}\right)\left(z_{i \nu}-z_{j \nu}\right)}{\eta_{i j}^{2}}\right)\right]\left(\alpha_{1} \xi_{i j}^{2}+\beta_{1} \zeta_{i j}^{2}\right)\right] \\
& -\frac{G^{2}}{c^{4}} \sum_{j \neq i} \sum_{k \neq i, j} m_{j} m_{k} \iint d \lambda_{j} d \lambda_{k} \\
& {\left[\delta\left(\rho_{i j}\right) \delta\left(\rho_{j k}\right)\left(\frac{1}{2} a_{0} \xi_{j k}\left(\dot{z}_{j \mu} \dot{z}_{k \nu}+\dot{z}_{k \mu} \dot{z}_{j \nu}\right)+b_{0} \zeta_{j} \zeta_{k} \eta_{\mu \nu}+c_{0} \zeta_{j} \dot{z}_{k \mu} \dot{z}_{k \nu}\right)\right.} \\
+ & \delta\left(\rho_{j k}\right) \delta\left(\rho_{k i}\right)\left(\frac{1}{2} a_{0} \xi_{j k}\left(\dot{z}_{k \mu} \dot{z}_{j \nu}+\dot{z}_{j \mu} \dot{z}_{k \nu}\right)+b_{0} \zeta_{j} \zeta_{k} \eta_{\mu \nu}+c_{0} \zeta_{k} \dot{z}_{j \mu} \dot{z}_{j \nu}\right) \\
+ & \left.\delta\left(\rho_{k i}\right) \delta\left(\rho_{i j}\right)\left(\frac{1}{2} a_{0} \xi_{j k}\left(\dot{z}_{k \mu} \dot{z}_{j \nu}+\dot{z}_{j \mu} \dot{z}_{k \nu}\right)+\left(b_{0} \zeta_{k} \zeta_{j}+c_{0} \xi_{j k}^{2}\right) \eta_{\mu \nu}\right)\right]
\end{aligned}
$$

For the case of a test particle (the mass of which can be neglected) in the presence of $N$ particles with non-negligible masses $m_{i}(i=1, \ldots, N)$ the above expression simplifies to the following:

$$
g_{\mu \nu}=\eta_{\mu \nu}-\frac{2 G}{c^{2}} \sum_{i} m_{i} \int d \lambda_{i} \delta\left(\left(z-z_{i}\right)^{2}\right)\left[\alpha_{0} \dot{z}_{i \mu} \dot{z}_{i \nu}+\beta_{0} \dot{z}_{i}^{2} \eta_{\mu \nu}\right]
$$




$$
\begin{aligned}
& -\frac{G^{2}}{c^{4}} \sum_{i} m_{i}^{2} \int d \lambda_{i} \frac{\delta\left(\left(z-z_{i}\right)^{2}\right)\left(\dot{z}_{i}^{2}\right)^{\frac{1}{2}}}{\left|\left(\dot{z}_{i}\left(z-z_{i}\right)\right)\right|}\left[\alpha_{1} \dot{z}_{i \mu} \dot{z}_{i \nu}+\beta_{1} \dot{z}_{i}^{2} \eta_{\mu \nu}\right] \\
& -\frac{G^{2}}{c^{4}} \sum_{i} \sum_{j \neq i} m_{i} m_{j} \iint d \lambda_{i} d \lambda_{j} \\
& {\left[\delta\left(\left(z-z_{i}\right)^{2}\right) \delta\left(\left(z_{i}-z_{j}\right)^{2}\right)\left(\frac{1}{2} a_{0}\left(\dot{z}_{i} \dot{z}_{j}\right)\left(\dot{z}_{i \mu} \dot{z}_{j \nu}+\dot{z}_{j \mu} \dot{z}_{i \nu}\right)+b_{0} \dot{z}_{i}^{2} \dot{z}_{j}^{2} \eta_{\mu \nu}+c_{0} \dot{z}_{i}^{2} \dot{z}_{j \mu} \dot{z}_{j \nu}\right)\right.} \\
& +\delta\left(\left(z_{i}-z_{j}\right)^{2}\right) \delta\left(\left(z-z_{j}\right)^{2}\right)\left(\frac{1}{2} a_{0}\left(\dot{z}_{i} \dot{z}_{j}\right)\left(\dot{z}_{i \mu} \dot{z}_{j \nu}+\dot{z}_{j \mu} \dot{z}_{i \nu}\right)+b_{0} \dot{z}_{i}^{2} \dot{z}_{j}^{2} \eta_{\mu \nu}+c_{0} \dot{z}_{j}^{2} \dot{z}_{i \mu} \dot{z}_{i \nu}\right) \\
& \left.+\delta\left(\left(z-z_{j}\right)^{2}\right) \delta\left(\left(z-z_{i}\right)^{2}\right)\left(\frac{1}{2} a_{0}\left(\dot{z}_{i} \dot{z}_{j}\right)\left(\dot{z}_{i \mu} \dot{z}_{j \nu}+\dot{z}_{j \mu} \dot{z}_{i \nu}\right)+\left(b_{0} \dot{z}_{i}^{2} \dot{z}_{j}^{2}+c_{0}\left(\dot{z}_{i} \dot{z}_{j}\right)^{2}\right) \eta_{\mu \nu}\right)\right]
\end{aligned}
$$

The first Post-Newtonian approximation

The equations of motion (69) involve multiple times. The force acting on mass $i$ depends on the state of motion of particle $i$ at time $t$ and, to account for the time needed for the transmission of the interactions, on the states of motion of the remaining $N-1$ particles at the past and future times $t_{j}^{(i, s)}(j \neq i, s=-,+)$ and also on $t_{k}^{(j, s)}(k \neq i, j, s=-,+)$.

Using Taylor series expansions involving the particles' present motions at time $t$, one can rewrite the equations (69) using just the one time variable $t$ [56]. We use series expansions up to terms of second order $\left(\frac{v^{2}}{c^{2}}\right)$ (first Post-Newtonian approximation $(1 \mathrm{PN})$ ).

From the definition (5) it follows that:

$$
\begin{gathered}
d \lambda_{i}=\frac{c d t\left(1-\frac{v_{i}^{2}}{c^{2}}\right)^{\frac{1}{2}}}{\zeta_{i}^{\frac{1}{2}}} \\
d \lambda_{j}=\frac{c d t_{j}\left(1-\frac{v_{j}^{2}\left(t_{j}\right)}{c^{2}}\right)^{\frac{1}{2}}}{\zeta_{j}^{\frac{1}{2}}}
\end{gathered}
$$

The Dirac delta function can be expressed as follows [10]:

$$
\delta\left(c^{2}\left(t-t_{j}\right)^{2}-\left(\vec{r}_{i}-\vec{r}_{j}\right)^{2}\right)=\frac{1}{2 c}\left(\frac{\delta\left(t_{j}-t_{j}^{(i,-)}\right)}{\left(R_{i j}^{r e t}-\frac{\left(\vec{R}_{i j}^{r e t} \vec{v}_{j}^{(i,-)}\right)}{c}\right)}+\frac{\delta\left(t_{j}-t_{j}^{(i,+)}\right)}{\left(R_{i j}^{a d v}+\frac{\left(\vec{R}_{i j}^{a d v} \vec{v}_{j}^{(i,+)}\right)}{c}\right)}\right)
$$

In (80),$t_{j}^{(i, s)}(s=-,+)$ are the two roots of the equation:

$$
c^{2}\left(t-t_{j}\right)^{2}-\left(\vec{r}_{i}(t)-\vec{r}_{j}\left(t_{j}\right)\right)^{2}=0
$$

and,

$$
\begin{aligned}
& R_{i j}^{r e t}=c\left(t-t_{j}^{(i,-)}\right) \\
& R_{i j}^{a d v}=c\left(t_{j}^{(i,+)}-t\right)
\end{aligned}
$$




$$
\begin{aligned}
\vec{R}_{i j}^{r e t} & =\vec{r}_{i}-\vec{r}_{j}^{(i,-)} \\
\vec{R}_{i j}^{a d v} & =\vec{r}_{i}-\vec{r}_{j}^{(i,+)}
\end{aligned}
$$

$t-t_{j}^{(i,-)}$ is the time it takes for a signal to travel forward in time at the speed of light from particle $j$ to particle $i$.

$t_{j}^{(i,+)}-t$ is the time it takes for a signal to travel backward in time at the speed of light from particle $j$ to particle $i$.

To terms of second order we can write:

$$
\begin{aligned}
& \vec{r}_{i}-\vec{r}_{j}^{(i,-)} \approx \vec{r}_{i j}+\vec{v}_{j} \frac{r_{i j}}{c}+\vec{v}_{j} \frac{\left(\vec{r}_{i j} \vec{v}_{j}\right)}{c^{2}}-\vec{a}_{j} \frac{r_{i j}^{2}}{2 c^{2}}, \\
& \vec{r}_{i}-\vec{r}_{j}^{(i,+)} \approx \vec{r}_{i j}-\vec{v}_{j} \frac{r_{i j}}{c}+\vec{v}_{j} \frac{\left(\vec{r}_{i j} \vec{v}_{j}\right)}{c^{2}}-\vec{a}_{j} \frac{r_{i j}^{2}}{2 c^{2}}, \\
& \vec{v}_{j}^{(i,-)} \approx \vec{v}_{j}-\vec{a}_{j} \frac{r_{i j}}{c}, \\
& \vec{v}_{j}^{(i,+)} \approx \vec{v}_{j}+\vec{a}_{j} \frac{r_{i j}}{c} .
\end{aligned}
$$

From (81,89), we find (to terms of second order):

$$
\begin{gathered}
\frac{\left(1-\frac{v_{j}^{(i,-) 2}}{c^{2}}\right)^{\frac{1}{2}}}{\left(R_{i j}^{r e t}-\frac{\left(\vec{R}_{i j}^{r e t} \vec{v}_{j}^{(i,-)}\right)}{c}\right)} \approx \frac{1}{r_{i j}}\left(1-\frac{\left(\vec{n}_{i j} \vec{v}_{j}\right)^{2}}{2 c^{2}}-\frac{\left(\vec{r}_{i j} \vec{a}_{j}\right)}{2 c^{2}}\right) \\
\frac{\left(1-\frac{v_{j}^{(i,+) 2}}{c^{2}}\right)^{\frac{1}{2}}}{\left(R_{i j}^{a d v}+\frac{\left(\vec{R}_{i j}^{a d v} \vec{v}_{j}^{(i,+)}\right)}{c}\right)} \approx \frac{1}{r_{i j}}\left(1-\frac{\left(\vec{n}_{i j} \vec{v}_{j}\right)^{2}}{2 c^{2}}-\frac{\left(\vec{r}_{i j} \vec{a}_{j}\right)}{2 c^{2}}\right)
\end{gathered}
$$

From the definitions (3, 4, 5) and (39, 40), it is not difficult to see that, to terms of second order, we can write:

$$
\begin{gathered}
\frac{\xi_{i j}}{\zeta_{i j}} \approx 1+\frac{v_{i}^{2}}{2 c^{2}}+\frac{v_{j}^{2}}{2 c^{2}}-\frac{\left(\vec{v}_{i} \vec{v}_{j}\right)}{c^{2}} \\
\eta_{j i}^{(i,-)} \approx r_{i j}\left(1+\frac{\left(\vec{n}_{i j} \vec{v}_{j}\right)^{2}}{2 c^{2}}+\frac{\left(\vec{r}_{i j} \vec{a}_{j}\right)}{2 c^{2}}\right), \\
\eta_{j i}^{(i,+)} \approx-r_{i j}\left(1+\frac{\left(\vec{n}_{i j} \vec{v}_{j}\right)^{2}}{2 c^{2}}+\frac{\left(\vec{r}_{i j} \vec{a}_{j}\right)}{2 c^{2}}\right), \\
\eta_{i j}^{(i,-)} \approx-r_{i j}\left(1-\frac{\left(\vec{n}_{i j} \vec{v}_{i}\right)}{c}+\frac{\left(\vec{n}_{i j} \vec{v}_{j}\right)}{c}+\frac{\left(\vec{v}_{i}-\vec{v}_{j}\right)^{2}}{2 c^{2}}+\frac{\left(\vec{n}_{i j} \vec{v}_{j}\right)^{2}}{2 c^{2}}-\frac{\left(\vec{r}_{i j} \vec{a}_{j}\right)}{2 c^{2}}\right),
\end{gathered}
$$




$$
\eta_{i j}^{(i,+)} \approx r_{i j}\left(1+\frac{\left(\vec{n}_{i j} \vec{v}_{i}\right)}{c}-\frac{\left(\vec{n}_{i j} \vec{v}_{j}\right)}{c}+\frac{\left(\vec{v}_{i}-\vec{v}_{j}\right)^{2}}{2 c^{2}}+\frac{\left(\vec{n}_{i j} \vec{v}_{j}\right)^{2}}{2 c^{2}}-\frac{\left(\vec{r}_{i j} \vec{a}_{j}\right)}{2 c^{2}}\right) .
$$

In (86- 96), $\vec{r}_{i j}=\vec{r}_{i}-\vec{r}_{j}$ is the relative position of particle $i$ with respect to particle $j, \vec{n}_{i j} \equiv \frac{\vec{r}_{i j}}{r_{i j}}, \vec{v}_{i}$ is the velocity of particle $i$, and $\vec{v}_{j}, \vec{a}_{j}$ the velocity and the acceleration of particle $j$. All these quantities are given at time $t$.

From (25) and (67), at the first Post-Minkowskian order, for the solutions of the equations of motion we obtain:

$$
\zeta_{i}=1+\frac{2 G}{c^{2}} \sum_{j \neq i} m_{j} \int d \lambda_{j} \delta\left(\rho_{i j}\right) \zeta_{j}\left(\alpha_{0} \frac{\xi_{i j}^{2}}{\zeta_{i j}^{2}}+\beta_{0}\right)
$$

Now, substituting (79,80) and (90,91,92) into (97), to terms of second order, for the solutions of the equations of motion we can write:

$$
\zeta_{i} \approx 1+\frac{2 G}{c^{2}}\left(\alpha_{0}+\beta_{0}\right) \sum_{j \neq i} \frac{m_{j}}{r_{i j}} .
$$

Substituting (78- -96) and (98) into (69- 75), we find the equations of motion to terms of second order (in $\frac{v^{2}}{c^{2}}$ ) (first Post-Newtonian approximation):

$$
\begin{aligned}
& \vec{a}_{i}+G\left(\alpha_{0}+\beta_{0}\right) \sum_{j \neq i} \frac{m_{j}}{r_{i j}^{2}} \vec{n}_{i j}+\frac{v_{i}^{2}}{c^{2}} \vec{a}_{i}+\frac{\left(\vec{v}_{i} \vec{a}_{i}\right)}{c^{2}} \vec{v}_{i}+\frac{2 G \alpha_{0}}{c^{2}} \vec{a}_{i} \sum_{j \neq i} \frac{m_{j}}{r_{i j}}-\frac{G}{2 c^{2}}\left(3 \alpha_{0}-\beta_{0}\right) \sum_{j \neq i} \frac{m_{j}}{r_{i j}} \vec{a}_{j} \\
& -\frac{G}{2 c^{2}}\left(\alpha_{0}+\beta_{0}\right) \sum_{j \neq i} \frac{m_{j}}{r_{i j}} \vec{n}_{i j}\left(\vec{n}_{i j} \vec{a}_{j}\right)+\frac{G}{c^{2}} \sum_{j \neq i} \frac{m_{j}}{r_{i j}^{2}} \vec{n}_{i j}\left(\alpha_{0}\left(v_{i}^{2}+v_{j}^{2}-2\left(\vec{v}_{i} \vec{v}_{j}\right)\right)-\frac{3}{2}\left(\alpha_{0}+\beta_{0}\right)\left(\vec{n}_{i j} \vec{v}_{j}\right)^{2}\right) \\
& +\frac{G}{c^{2}}\left(\beta_{0}-\alpha_{0}\right) \vec{v}_{i} \sum_{j \neq i} \frac{m_{j}}{r_{i j}^{2}}\left(\left(\vec{n}_{i j} \vec{v}_{i}\right)-\left(\vec{n}_{i j} \vec{v}_{j}\right)\right)+\frac{G}{c^{2}} \sum_{j \neq i} \frac{m_{j}}{r_{i j}^{2}} \vec{v}_{j}\left(2 \alpha_{0}\left(\vec{n}_{i j} \vec{v}_{i}\right)-\left(\alpha_{0}-\beta_{0}\right)\left(\vec{n}_{i j} \vec{v}_{j}\right)\right) \\
& +\frac{G^{2} m_{i}}{c^{2}}\left(\left(\alpha_{0}+\beta_{0}\right)^{2}+\alpha_{1}+\beta_{1}\right) \sum_{j \neq i} \frac{m_{j}}{r_{i j}^{3}} \vec{n}_{i j}+\frac{G^{2}}{c^{2}}\left(2\left(\alpha_{0}+\beta_{0}\right)^{2}+\alpha_{1}+\beta_{1}\right) \sum_{j \neq i} \frac{m_{j}^{2}}{r_{i j}^{3}} \vec{n}_{i j} \\
& +\frac{G^{2}}{c^{2}} \sum_{j \neq i} \sum_{k \neq i, j} \frac{m_{j} m_{k}}{r_{i j}^{2}} \vec{n}_{i j}\left(\frac{1}{r_{i k}}\left(2\left(\alpha_{0}+\beta_{0}\right)^{2}+a_{0}+b_{0}+c_{0}\right)+\frac{1}{r_{j k}}\left(\left(\alpha_{0}+\beta_{0}\right)^{2}+a_{0}+b_{0}+c_{0}\right)\right) \\
& =0 .
\end{aligned}
$$

Complete agreement with the equations of motion of General Relativity [57, 58], at the first Post-Newtonian order, is achieved if:

$$
\begin{gathered}
\alpha_{0}=2, \\
\beta_{0}=-1, \\
\alpha_{1}+\beta_{1}=-2, \\
a_{0}+b_{0}+c_{0}=-2 .
\end{gathered}
$$


Conclusions

We have obtained Lorentz invariant equations of motion describing the gravitational interactions of a system consisting of $N$ point masses. The equations are derived explicitly from a Lorentz invariant action. Contrary to General Relativity, which is a field theory, the model presented here is a relativistic action-at-a-distance description (the interactions are not mediated by a field). We have shown that the equations of motion for $N$ point masses agree with those of General Relativity at the first Post-Newtonian approximation (1PN). Agreement with General Relativity for the $N$ body problem at orders beyond 1.5PN has not been established. The model presented is in agreement with General Relativity for the onebody case, at all orders. At the first Post-Minkowskian approximation our model reduces to the model of Havas and Golberg [50, 51], which is known to be in agreement with General Relativity in this approximation. Due to this agreement, gravitational radiation effects in our model begin to appear at the $2.5 \mathrm{PN}$ order $\left(\frac{v^{5}}{c^{5}}\right)[59,60]$.

\section{References}

[1] K. Schwarzschild, Gottingen Nachrichten 128, 132 (1903).

[2] H. Tetrode, Z. Phys. 10, 317 (1922).

[3] A. D. Fokker, Physica 12, 145 (1932).

[4] J. A. Wheeler and R. P. Feynman, Rev. Mod. Phys. 17, 157 (1945).

[5] J. A. Wheeler and R. P. Feynman, Rev. Mod. Phys. 21, 425 (1949).

[6] R.A. Moore, T.C. Scott and M.B. Monagan, Phys. Rev. Lett. 59, 525 (1987).

[7] F. Hoyle and J.V. Narlikar, Lectures on Cosmology and Action at a Distance Electrodynamics, (World Scientific, 1996).

[8] A. Schild, Phys. Rev. 131, 2762 (1963).

[9] D.J. Louis-Martinez, Phys. Lett. A320, 103 (2003).

[10] A. O. Barut, Electrodynamics and Classical Theory of Fields and Particles, (Dover Publications Inc, New York, 1980).

[11] J.W. Dettman and A. Schild, Phys. Rev. 95, 1057 (1954).

[12] C.M. Andersen and H.C. von Baeyer, Ann. Phys. (N.Y.) 60, 67 (1970).

[13] A. Schild, Ann. Phys. (N.Y.) 93, 88 (1975).

[14] P. Ramond, Phys. Rev. D7, 449 (1973).

[15] A. Katz, J. Math. Phys. 10, 1929 (1969); 10, 2215 (1969).

[16] A. Degasperis, Phys. Rev. D3, 273 (1971).

[17] J.M. Cornwall, Nucl. Phys. B128, 75 (1977).

[18] A. Rivacoba, Nuovo Cim. B84, 35 (1984). 
[19] J. Weiss, J. Math. Phys. 27, 1015 (1986).

[20] M. Kalb and P. Ramond, Phys. Rev. D9, 2273 (1974).

[21] B. Jensen and U. Lindstrom Phys. Lett. B398, 83 (1997).

[22] R. N. Hill, J. Math. Phys. 8, 201 (1967).

[23] E.H. Kerner, J. Math. Phys. 9, 222 (1968).

[24] Ph. Droz-Vincent, Lett. Nouvo Cimento 1, 839 (1969)

[25] L. Bel and X. Fustero, Ann. Inst. H. Poincare A 25, 411 (1976).

[26] I. T. Todorov, in Relativistic Action at a Distance: Classical and Quantum Aspects, Ed. by J. Llosa, Lectures Notes in Physics 162, Springer-Verlag (1982).

[27] L. P. Horwitz and F. Rohrlich, Phys. Rev. D24, 1528 (1981).

[28] H. Sazdjian, Ann. of Phys. 136, 136 (1981).

[29] G. Longhi, D. Dominici, J. Gomis and J. A. Lobo in Relativistic Action at a Distance: Classical and Quantum Aspects, Ed. by J. Llosa, Lectures Notes in Physics 162, Springer-Verlag (1982).

[30] J. Bijtebier, Nucl. Phys. A696, 581 (2001).

[31] Ph. Droz-Vincent, Int. J. Theor. Phys. 42, 1809 (2003).

[32] H. Poincare, Rend. Circ. Mat., Palermo, 21, 166 (1906).

[33] W. De Sitter, M. N. Roy. Astron. Soc. 71, 388 (1911).

[34] A. N. Whitehead, The Principle of Relativity (Cambridge Univ. Press, Cambridge, 1922).

[35] A. S. Eddington, The Mathematical Theory of Relativity (Cambridge Univ. Press, 1924).

[36] J. L. Synge, Proc. Roy. Soc. London A211, 303 (1952).

[37] J. Dyer and A. Schild, Journal of Mathematical Analysis and Applications 4, 328 (1962).

[38] F. Hoyle and J.V. Narlikar, Proc. R. Soc., Ser. A 282, 191 (1964).

[39] S. W. Hawking, Proc. R. Soc. Lond. A286, 313 (1965).

[40] P.C.W. Davis, Nature 228, 270 (1970).

[41] E. G. Harris, Am. J. Phys. 49, 1051 (1981).

[42] Yu. S. Vladimirov and A. Yu. Turygin, Theory of Direct Interparticle Interaction (in Russian, Energoatomizdat, Moscow, 1986).

[43] A. Yu. Turygin, General Relativity and Gravitation 18, 333 (1986). 
[44] R.P. Gaida, Yu. B. Klyuchkovskii and V.I. Tretyak, Russian Physics Journal 33, 40 (1990).

[45] G. N. Afanasiev and R. A. Asanov, Physics of Particles and Nuclei 27, 296 (1996).

[46] Yu. S. Vladimirov, Gravitation and Cosmology, 14, 41 (2008).

[47] G. J. Whitrow and G. E. Morduch, in Vistas in Astronomy, 6, 1, A. Beer, ed. (Pergamon, New York, 1965).

[48] G. Gibbons and C. M. Will, Studies In History and Philosophy of Science Part B: Studies In History and Philosophy of Modern Physics 39, 41 (2008).

[49] D. Louis-Martinez, Phys. Lett. B 632, 733 (2006).

[50] J.N. Golberg, in Gravitation: An Introduction to Current Research, L. Witten, ed. (Wiley, New York, 1962).

[51] P. Havas and J.N. Golberg, Phys. Rev. 128, 398 (1962).

[52] J.L. Anderson, Principles of Relativity Physics (Academic Press, New York, 1967).

[53] J. Friedman and K. Uryu, Phys. Rev. D73, 104039 (2006).

[54] D.J. Louis-Martinez, Found. Phys. 42, 215 (2012).

[55] S. Weinberg, Gravitation and Cosmology (Wiley, 1972).

[56] D.J. Louis-Martinez, Phys. Lett. A 364, 93 (2007).

[57] A. Einstein, L. Infeld and B. Hoffmann, Ann. Math. 39, 65 (1938).

[58] L. D. Landau and E. M. Lifshitz, The Classical Theory of Fields (4th revised English edition), Butterworth- Heinenann, Oxford (1996).

[59] S.F. Smith and P. Havas, Phys. Rev. 138, B495 (1965).

[60] M. Walker and C.M. Will, The Astrophysical Journal 242, L129 (1980). 